CAE Working Paper \#06-14

Flexibility of Choice versus Reduction of Ambiguity

by

Ani Guerdjikova

and

Alexander Zimper

August 2006 


\title{
Flexibility of choice versus reduction of ambiguity*
}

\author{
Ani Guerdjikova ${ }^{\dagger} \quad$ Alexander Zimper
}

August 15, 2006

\begin{abstract}
This paper explores the problem of a social planner willing to improve the welfare of individuals who are unable to compare all available alternatives. The optimal decision trades off the individuals' desire for flexibility versus their aversion towards ambiguous choice situations. We introduce an axiom system that formalizes this idea. Our main result characterizes the preference maximizing opportunity set. It is a maximal set that consists of mutually comparable alternatives. It also has the property that it maximizes the sum of the distances between its ordered elements for some appropriate metric imposed on the set of possible choices.
\end{abstract}

Keywords: incomplete preferences, ambiguity, flexibility of choice, opportunity sets, uncertainty

JEL Classification Numbers: D69, D81.

${ }^{*}$ We thank Kaushik Basu, Larry Blume, Ted O'Donoghue, Eric Danan, Juergen Eichberger, Hans Haller, Juliane Hiesgen, Alexander Ludwig, Klaus Nehring, Klaus Ritzberger and David Schmeidler for helpful comments and suggestions. We are especially grateful to an anonymous referee who helped a lot to improve the consistency of our approach. Both authors gratefully acknowlege financial support from the Deutsche Forschungsgemeinschaft, Sonderforschungsbereich 504, University of Mannheim.

${ }^{\dagger}$ Cornell University, Department of Economics, Uris Hall 462, Ithaca, NY 14853, USA. Email: ag334@cornell.edu

${ }^{\ddagger}$ Corresponding author. School of Economics, Faculty of Commerce, University of Cape Town, Private Bag, Rondebosch, 7701, Cape Town, RSA. Email: zimper@bigfoot.com. 


\section{Introduction}

The standard result that rational decision-makers should prefer larger opportunity sets is implied by the assumption of a preference relation that admits the comparison of all possible alternatives at no cost. Although the existence of such a preference relation may be unquestionable in situations where there are few alternatives that are easily comparable, its assumption strikes us as quite counterintuitive in situations in which a vast amount of alternatives must be compared according to multiple criteria. In such situations, a decision-maker might prefer to trade-off a larger opportunity set against a smaller one that includes fewer incomparable alternatives.

In this paper, we model preferences over opportunity sets under the assumptions that, first, the decision-maker is unable to compare all available alternatives, either ex ante or ex post; second, the decision-maker perceives any ex post restriction of his ex ante chosen menu through forces of nature as possible. The first assumption is captured by an incomplete preference relation over alternatives, which is known in advance and, therefore, deterministic. Combined with a strong aversion of the decision-maker against making choices between incomparable alternatives - which we call strong ambiguity aversion - incomplete preferences imply that the decision-maker will express preferences for smaller opportunity sets, preferring menus that do not contain incomparable alternatives. In contrast, the second assumption forces the individual to express preferences for flexibility: since nature may eliminate the best element out of the initially chosen menu, it is preferable to choose an opportunity set that also contains the second best alternative given the restrictions imposed by nature.

As our main contribution we explore the question of how a welfare-maximizing social planner optimally restricts opportunity sets. For this purpose we impose axioms on preferences over opportunity sets and derive a characterization theorem stating that

- Any preference maximizing opportunity set must be a maximizer of a utility function that sums up the distances between its ordered elements for some appropriate metric imposed on the set of alternatives;

- Conversely, if an opportunity set maximizes a utility function that sums up the distances between its ordered elements for an arbitrary metric, then this opportunity set is considered optimal for some preference ordering satisfying our axioms.

Our axiom system models the trade-off between preferences for flexibility and ambiguity aversion in a very special way. The decision-maker we have in mind considers any set containing incomparable alternatives to be inferior to some set on which the preference relation is complete. Hence, sets containing incomparable alternatives can never be 
an optimal choice. Moreover, the incompleteness of preferences, which is not resolved ex post, does not allow for a meaningful representation of preferences by an expected utility functional. The preference for flexibility further implies that the decision-maker prefers a maximal set out of those on which the preference relation is complete.

To give an example: Consider a college graduate who has to decide on the set of PhD programs to which to apply. On the one hand, there is the worst case scenario where the student is not admitted to any $\mathrm{PhD}$ program he had applied to. On the other hand, the student might be simultaneously admitted to a large number of $\mathrm{PhD}$ programs to the effect that he has problems to make up his mind. Suppose, for instance, that any of his alternatives are characterized by two sets of features:

$$
\begin{aligned}
& \Lambda:=\text { prestige } \times \text { tuition } \times \text { scholarship } \\
& \Gamma:=\text { pressure to perform } .
\end{aligned}
$$

While the student may find it easy to compare different values of $\lambda \in \Lambda$, let us assume that he encounters difficulties to compare different values of $\gamma \in \Gamma$, i.e., the pressure to perform. Possible reasons for this might be that the prospective $\mathrm{PhD}$ student is unsure about his own abilities or that he is ambivalent towards working under pressure: on the one hand, he enjoys proving himself in a competitive environment, on the other hand, he would physically suffer from the stress. As a consequence, it might be difficult for him to decide between $\mathrm{PhD}$ programs that differ in their pressure to perform. When such a student decides on the set of PhD programs to which to apply, he must therefore take into account the following trade-off: while applying to a large number of $\mathrm{PhD}$ programs increases the chances of being admitted, it also increases the likelihood of being caught in an ambiguous choice situation where he is faced with several incomparable offers to choose from.

For the above example, our axiomatic approach suggests that a student who is ambiguity averse will choose to apply to a set of $\mathrm{PhD}$ programs which are well ordered with respect to his preferences and will avoid sets containing incomparable alternatives. Even more relevantly, our approach also suggests that there might be positive welfare effects of real-life institutions that effectively restrict choice-sets. For example, in many countries (e.g., Bulgaria, Germany) the application to colleges is centralized whereby prospective students are asked to list the colleges they wish to apply in the order of their preferences. Usually, there is no limit on how many choices a prospective student is allowed to make, 
but the ordering has to be unambiguous. This institution, hence, forces applicants to choose a set of comparable colleges. After the ranking of the applicants has been determined, assigning to each prospective student the best choice according to his preferences which is consistent with the ranking is optimal from the point of view of each student. ${ }^{1}$

The remainder of the paper proceeds as follows. Section 2 discusses the relationship of our approach to the existing psychologic and economic literature. In section 3, we define our central concepts of strong ambiguity aversion and flexibility of choice. Section 4 introduces our main technical assumption of cancellation and presents an additive representation result. Our main result is derived in section 5 where we characterize preference maximizing opportunity sets as maximizers of a specific utility representation. In section 6 we illustrate our formal results for a specific class of preferences over alternatives exhibiting a comparable as well as an incomparable component. We conclude in section 7 .

\section{Related literature}

Our approach is motivated by the psychological literature on choice-behavior while our formalism is closely related to models in the economic literature on preferences for flexibility and preferences for commitment. Furthermore, we contribute to the decisiontheoretic literature on behavioral consequences of incomplete preferences.

The psychological literature provides evidence for the trade-off between larger and smaller choice sets. Larger sets are found to have motivating power on individual behavior and to reduce anxiety. Smaller sets instead reduce the complexity of decisions and ensure that alternatives can be meaningfully compared. While experiments, in which the number of alternatives is relatively small (2-6), support the hypothesis that more alternatives improve the well-being of the individual (cf. Deci, 1975, 1981; Deci and Ryan, 1985), a significant increase in the number of possible alternatives (up to 30) may make the individual worse off. Subjects in experiments tend to make suboptimal decisions, delay making choices and feel regret over the choices actually made (see Shafir, Simonson and Tversky (1993), Kahneman and Tversky (1984), Iyengar and Lepper (2000), (2002)). Most of this literature is concerned with choices that do not significantly affect the well-being of an individual (e.g. choices among different types of jam or chocolate). Surprisingly, similar effects also seem to be present when people are facing decisions of significant importance, as, e.g., the choice of a retirement saving plan. Iyengar, Jiang

\footnotetext{
${ }^{1}$ Using the Gale-Shapley (1962) mechanism to match students with schools would insure that prospective students have no incentives to misrepresent their preferences.
} 
and Huberman (2004) demonstrate that employees are more willing to contribute to a pension scheme if less options are presented to them.

Some of the experiments (cf. Iyengar and Lepper, 2000) show that people tend to exhibit dynamic inconsistency: subjects in experiments prefer a larger choice set ex ante despite the regret they experience after the choice has been made. In face of this empirical evidence, our axiomatization of preferences over opportunity sets can be viewed as a model of a decision-maker who behaves sequentially rational (=behaviorally consistent in the terminology of Karni and Safra, 1990), anticipating the dissatisfaction resulting from a choice out of a large opportunity set and, therefore, wishes to restrict his opportunities ex ante. On the other hand, one might assume that bounded foresight is by large present in human decisions and view the model presented below as axiomatizing the decision of a social planner willing to correct the effects of boundedly rational behavior. Thaler and Sunstein (2003) argue in favor of such corrections termed "libertarian paternalism": a social planner must at times restrict the choices available to individuals, while still allowing for sufficient flexibility of choice.

In a seminal contribution to the economic theory on preferences over opportunity sets, Kreps (1979) considers a two-stage decision situation where the decision-maker chooses in period 1 some opportunity set from which he may pick, in period 2 , some alternative. In Kreps' approach the decision-maker exhibits preferences for flexibility because he is ex ante (period 1) uncertain about his ex post (period 2) preferences over alternatives. While Kreps obtains a representation result where some implicit states of the world appear as part of the representation — but not as part of the set-up for decision-making under uncertainty - Nehring (1999) describes preferences for flexibility in a Savage (1954) framework where states of the world are explicitly given in the set-up in order to capture uncertainty about future opportunity sets. Dekel, Lipman, and Rustichini (2001) extend Kreps' model by additionally allowing for preferences for commitment. Dekel, Lipman and Rustichini (2001) describe preferences over opportunity sets which trade-off a desire for flexibility against a desire for reducing the number of alternatives in opportunity sets, which is similar to our approach. Dekel, Lipman and Rustichini (2001) do not themselves develop possible explanations for a desire for commitment. They rather refer to existing approaches in the literature which explain a desire for commitment either by dynamic inconsistencies, e.g. Strotz (1954), Laibson (1997), so that a commitment may improve the strategic situation of an ex ante decision-maker who plays a game with his future selves, or by the desire to avoid temptations, (cf. Gul and Pesendorfer, 2001, 2004). For instance, a decision-maker of their model might have to choose a restaurant before knowing his preferences over different meals in advance. On the one hand, he would prefer to have a richer opportunity set, on the other hand, if 
he is, say, on a diet, he might express the desire to avoid the appearance of certain kinds of food on the menu which he considers unhealthy though tempting. However, once a particular restaurant is chosen, the preferences of the decision-maker become known and he selects the best alternative out of the menu.

Our approach differs from this literature on preferences over opportunity sets in several respects. First, there is no uncertainty in our model about the decision-maker's ex post preferences but rather we presume, similar to Nehring (1999), that there is uncertainty about the moves of nature which may effectively restrict the alternatives available to the decision-maker in period 2. As a consequence, we have a clear characterization of the state space that captures all aspects of uncertainty in our model. Unlike the models of Kreps (1979), Dekel, Lipman, and Rustichini (2001) and Gul and Pesendorfer (2001), in our model, the best alternative might not be uniquely defined in period 2 for each realized state of nature and each opportunity set chosen in period 1 . This is a direct consequence of the incompleteness of preferences over alternatives. As a consequence, we cannot proceed as in this literature where ex ante preferences over opportunity sets are aggregated from ex post preferences determining the best alternatives in each state of the world. On the other hand, we do not wish to simply circumvent this incompleteness problem by stipulating, as in Nehring (1999), that ex post preferences are defined over opportunity sets rather than over best alternatives belonging to those ex post realized opportunity sets. Our proposal instead applies the Savage (1954) framework in order to motivate our main axioms of flexibility of choice and strong ambiguity aversion whereby we consider states in which best alternatives exist. Nevertheless, the application of the Savage framework is not sufficient here in order to completely determine ex ante preferences over opportunity sets since comparisons of different consequences at the same state may be impossible. We, therefore, impose our main technical axiom, cancellation, directly on preferences over opportunity sets without referring to a sophisticated decision-maker who is able to compare the consequences of his actions across different states so that his preferences may reveal his likelihood considerations.

Our motivation for a desire for commitment by the wish to avoid ambiguous choice situations is new to the economic literature. As Gul and Pesendorfer (2001) — and unlike Strotz (1956), Laibson (1997) or Ludwig and Zimper (2006) — we do not presume dynamic inconsistencies. However, our motivation of preferences for commitment also differs greatly from Gul and Pesendorfer's temptation-driven preferences. Gul and Pesendorfer (2001) introduce an axiom called set betweenness, which implies temptationdriven preferences. This axiom requires that for any two sets $D \succeq E$,

$$
D \succeq D \cup E \succeq E
$$

This axiom is violated by our assumption of strong ambiguity aversion whenever the 
individual can not unambiguously evaluate his opportunities. To see this, suppose that each of the sets $D$ and $E$ contains only mutually comparable alternatives, but for each alternative $a$ in $D$ there is an alternative $b$ in $E$ such that $a$ and $b$ are incomparable. Then, under our assumption of strong ambiguity aversion, the decision-maker will strictly prefer each of the unambiguous sets $E$ and $D$ to their union. Dekel, Lipman, and Rustichini (2004) provide examples which cast doubt on the appropriateness of set betweenness to capture temptation-driven preferences and propose a different axiom, desire for commitment. This axiom requires that for every set $D$, there is an element $a \in D$ such that

$$
\{a\} \succeq D
$$

This axiom is inconsistent with the assumption of flexibility of choice, which we impose and which requires that larger unambiguous sets are preferred to smaller ones.

Finally, our approach contributes to the literature on incomplete preferences which tries to find behavioral implications of incomplete preferences and distinguish them from the case of indifference. Danan and Ziegelmeyer (2004) suggest that incompleteness of preferences can be traced back to lack of information about the alternatives to be compared. Under this interpretation, the decision-maker will prefer to postpone his decision and will strictly prefer the choice set consisting of two alternatives to each of the singleton sets. Similarly to Danan and Ziegelmeyer (2004), Eliaz and Ok (2006) also assume that indecisiveness is characterized by selecting multiple alternatives. In contrast to this literature, we take incompleteness of preferences as a persisting feature which does not - necessarily - depend on acquiring additional information. Furthermore, our framework allows a distinction between preferences for flexibility and incomplete preferences on the one hand and between incompleteness and indifference, on the other hand.

\section{$3 \quad$ Ambiguity averse preferences over opportunity sets}

The key to our axiomatic approach is the presumption of two different preference re-

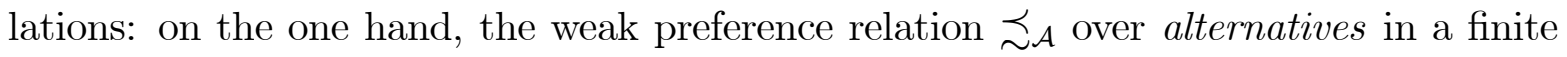
set $\mathcal{A}$; on the other hand, the weak preference relation $\succeq$ over opportunity sets, i.e., all non-empty subsets of $\mathcal{A}$. The decision-making process is as follows: in a first step a decision-maker is allowed to choose any subset $D$ of $\mathcal{A}$ according to $\succeq$. After that nature selects another subset of $\mathcal{A}$, say $E$, which constrains the initial choice of the individual. Hence, the decision-maker can now only choose among the alternatives contained in $D \cap E$, which he does according to his preferences $\precsim_{\mathcal{A}}$. We stipulate that there always exists a worst element $o \in D \cap E$. That is, neither the decision-maker's choice nor a 
move by nature can exclude $o$ from the alternatives being ex post available. A possible interpretation of $o$ would be the decision-maker's death ${ }^{2}$, which might always happen in the ex post situation: either by a move of nature or by the decision-maker's own hand. We assume that the decision-maker perceives any subset $E \subseteq \mathcal{A}$ such that $o \in E$ as a possible move of nature. Furthermore, we presume that the decision-maker's preferences $\precsim_{\mathcal{A}}$ over alternatives in $\mathcal{A}$ as well as preferences $\succeq$ over opportunity sets are identical in the ex ante and in the ex post situation, that is, before and after the individual learns how nature effectively restricts his possible choices.

Assumption 1: There exists a reflexive and transitive binary relation $\precsim \mathcal{A}$ over the elements in the set $\mathcal{A}$. $\mathcal{A}$ contains a worst alternative $o$, where $o \prec_{\mathcal{A}} a$, for all $a \in \mathcal{A}$.

By assumption 1, alternatives in $\mathcal{A}$ are only partially ordered with respect to the weak preference relation $\succsim$, that is, we allow for the possibility that there are alternatives $a, b \in \mathcal{A}$ such that not $a \precsim_{\mathcal{A}} b$ and not $b \precsim_{\mathcal{A}} a$. In that case we write $a A b$ and we say that "the individual has ambiguous preferences with respect to choosing $a$ over $b$ and vice versa". Thus, we formalize ambiguity as a reflexive binary relation over two alternatives with the interpretation that the individual is unable to say which alternative he (weakly) prefers. Ambiguity, according to our understanding, is therefore a very different concept than indifference: an individual who is indifferent between two alternatives, i.e., $a \sim b$, experiences no problem to simply choose one of these alternatives whereas an individual who has ambiguous preferences about these two alternatives, i.e., $a A b$, has an incredibly hard time to choose one alternative or the other.

Now focus on the weak preference relation $\succeq$ over opportunity sets. A preference statement $D \succeq E$ with $D, E \subseteq \mathcal{A}$ will be read by us as "the individual ex ante prefers the opportunity set $D$ to the opportunity set $E$." Recall that a chain is any non-empty subset of $\mathcal{A}$ that is totally ordered by $\precsim \mathcal{A}$. In our further discussion we concentrate on the subset of opportunity sets $\mathcal{D}_{o}$ defined as:

$$
\mathcal{D}_{o}=\{D \subseteq \mathcal{A} \mid o \in D\}
$$

Hence, $\mathcal{D}_{o}$ is the set of those opportunity sets which contain the worst alternative.

\footnotetext{
${ }^{2}$ Depending on the context there might be alternative - and less drastic - interpretations of $o$. E.g., in the example presented in the introduction, the worst alternative can be interpreted as not pursuing a doctoral degree, either because the applicant was not accepted to any of the universities, or because he decided to reject all offers.
} 
By $\mathcal{C} \subseteq \mathcal{D}_{o}$ we denote the set of all chains in $\mathcal{D}_{o}$ with respect to the order $\precsim \mathcal{A}_{\mathcal{A}}$ on $\mathcal{A}$. Note that all of these chains except the trivial one, $\{o\}$, contain at least two alternatives (this is the case, since $o$ is comparable to every element of $\mathcal{A}$ ). The generic element of $\mathcal{C}$ is either denoted by $h$ or by $\left(x_{1} \precsim \mathcal{A} \ldots \precsim_{\mathcal{A}} x_{n}\right)$ such that $x_{1} \neq \ldots \neq x_{n}$ and $x_{1} \equiv o$ for all $\left(x_{1} \precsim_{\mathcal{A}} \ldots \precsim_{\mathcal{A}} x_{n}\right) \in \mathcal{C}$.

Assumption 2: There exists a reflexive, transitive and complete binary relation $\succeq$ over all elements of $\mathcal{D}_{o}$.

By the following assumption we presume a structural richness of $\mathcal{A}$ which excludes uninteresting cases.

Assumption $3^{3}$ : For every $x \in \mathcal{A}$ there is some $y \in \mathcal{A}$ with $x \neq y$ such that $x \precsim \mathcal{A} y$ or $y \precsim \mathcal{A} x$.

Since nature may choose an arbitrary subset of $\mathcal{A}$, there exists a straightforward notion of relevant states of nature that capture the period 1 uncertainty in our model, namely all subsets of $\mathcal{A}$ containing the worst element $o$. The choice of an opportunity set in period 1 can be understood as a Savage-act that assigns to each state of nature as consequence the best alternative available for this given act and state of nature. However, under our assumptions, there may not exist a best alternative since the decision-maker may be confronted with ambiguous alternatives.

Consider, for example, the Savage-framework of a decision-situation where $\mathcal{A}=$ $\{o, a, b, c\}$ such that $a \prec_{\mathcal{A}} b$ and $a \prec_{\mathcal{A}} c$ but $b A c$. The interior cells of the following table depict the best alternative the decision-maker can choose ex post whereby "?" denotes an ambiguous choice situation in which the decision-maker is not able to say which of his alternatives at hand is best.

\footnotetext{
${ }^{3}$ Note that, besides assumption 3 , we assume no structure on the set $\mathcal{A}$. However, it is easy to show that every finite set $\mathcal{A}$ of cardinality $N$ together with the binary relations $\precsim$ and $A$ can be mapped on $\mathbb{R}^{N}$ in such way that $\precsim$ is represented by the natural order on vectors and incomparable alternatives are incomparable with respect to the natural ordering. (We thank Stefan Gerdjikov for this comment.)
} 
States of nature

$$
o \quad\{o, a\} \quad\{o, b\} \quad\{o, c\} \quad\{o, a, b\} \quad\{o, a, c\} \quad\{o, c, b\} \quad\{o, a, b, c\}
$$

Chosen opportunity sets

\begin{tabular}{|c|c|c|c|c|c|c|c|}
\hline$\{o, a\}$ & $O$ & $a$ & $O$ & $O$ & $a$ & $a$ & $o$ \\
\hline$\{o, b\}$ & $O$ & $o$ & $b$ & $O$ & $b$ & $o$ & $b$ \\
\hline$\{o, c\}$ & $O$ & $O$ & $O$ & $c$ & $O$ & $c$ & $c$ \\
\hline$\{o, a, b\}$ & $O$ & $a$ & $b$ & $O$ & $b$ & $a$ & $b$ \\
\hline$\{o, a, c\}$ & $O$ & $a$ & $O$ & $c$ & $a$ & $c$ & $c$ \\
\hline$\{o, b, c\}$ & $O$ & $o$ & $b$ & $c$ & $b$ & $c$ & $?$ \\
\hline$\{o, a, b, c\}$ & $O$ & $a$ & $b$ & $c$ & $b$ & $c$ & $?$ \\
\hline
\end{tabular}

In what follows we introduce our two central axioms - strong ambiguity aversion and flexibility of choice — which will effectively narrow down the possible candidates for preference-maximizing opportunity sets.

Definition: We say that the preference relation $\succeq$ over opportunity sets satisfies strong ambiguity aversion if and only if, for all $D \in \mathcal{D}_{o}$,

$$
D \succ D \cup\{b\}
$$

if there is some $a \in D$ such that $a A b, b \in \mathcal{A}$.

This definition of strong ambiguity aversion formalizes our central idea that individuals feel strictly more comfortable when there are less possibilities to end up in an ambiguous choice situation. In the above example, the Savage-acts $\{o, b, c\}$ and $\{o, a, b, c\}$ cannot be preference-maximizing whenever strong ambiguity aversion holds since we have, e.g.,

$$
\begin{aligned}
\left(o \prec_{\mathcal{A}} b\right) & \succ\left(o \prec_{\mathcal{A}} b\right) \cup\{c\} \text { and } \\
\left(o \prec_{\mathcal{A}} a \prec_{\mathcal{A}} b\right) & \succ\left(o \prec_{\mathcal{A}} a \prec_{\mathcal{A}} b\right) \cup\{c\} .
\end{aligned}
$$

To see that strong ambiguity aversion is indeed a rather strong assumption consider the following example (suggested to us by an anonymous referee): Suppose $x_{2} \precsim \mathcal{A} \ldots \precsim \mathcal{A}$ $x_{50}$ and $y \prec_{\mathcal{A}} x_{j}$ for $j \in\{3, \ldots, 50\}$ whereas $x_{2} A y$. By strong ambiguity aversion, the opportunity set $\left\{o, x_{2}, \ldots, x_{50}\right\}$ must be strictly preferred to the opportunity set $\left\{o, y, x_{2}, \ldots, x_{50}\right\}$. This is despite the fact that the ambiguity of the latter set seems to be negligible since ex ante there is a vast number of alternatives that are strictly preferred to 
the alternative $y$. Since the individual ex ante perceives any element of $\mathcal{D}_{o}$ as a possible reality in the ex post situation, it is plausible that he may strictly prefer the opportunity set $\left\{o, x_{2}, \ldots, x_{50}\right\}$ to $\left\{o, y, x_{2}, \ldots, x_{50}\right\}$ in order to avoid the unpleasant choice between $y$ and $x_{2}$ if nature chooses $\left\{o, y, x_{2}\right\}$. On the other hand, however, observe that the opportunity set $\left\{o, x_{2}, \ldots, x_{50}\right\}$ would leave the individual with the worst alternative, $o$, if nature chooses $\{o, y\}$. Since any alternative is, by assumption 1 , strictly better than $o$, the opportunity set $\left\{o, y, x_{2}, \ldots, x_{50}\right\}$ would have been, in retrospect, more favorable for that particular move of nature. Strong ambiguity aversion therefore describes decisionmakers who would like to avoid ambiguous choice situations rather than guaranteeing the existence of favorable alternatives.

The assumption of strong ambiguity aversion - as extreme as it is - has yet another advantage. In our present approach, we take the preference relation on the set $\mathcal{A}$ as a primitive which is known both to the decision-maker and the analyst. In an experimental setting, however, both relations $\precsim \mathcal{A}$ and $\succeq$ are subjective and have to be derived from observable choices. Thus, whenever decision-makers express preferences $\precsim \mathcal{A}$ that comply with our assumption of strong ambiguity aversion, we are able to uniquely and completely identify the incomparable pairs of alternatives. Our approach therefore entails a way of testing for incomplete preferences $\succeq$ which is alternative to the existing approaches by Danan and Ziegelmeyer (2004) and by Eliaz and Ok (2006).

Definition: We say that the preference relation $\succeq$ over opportunity sets satisfies flexibility of choice if and only if, for all non-degenerate chains $h:=\left(x_{1} \precsim_{\mathcal{A}} \ldots \precsim_{\mathcal{A}} x_{n}\right) \in$ C

$$
h \succeq h \backslash\left\{x_{j}\right\}
$$

for all $j \in\{2, \ldots, n\}$.

Flexibility of choice has a straightforward justification by our assumption that the individual is ex ante uncertain about his possible choices in the ex post situation whereby he perceives any subset of $\mathcal{A}$ containing $o$ as a possible reality in the ex post situation. For instance, the opportunity set $\{o, a, b\}$ weakly dominates in our example the opportunity sets $\{o, a\}$ and $\{o, b\}$ since it gives in each state of the world a consequence from the set $\mathcal{A}$ that is at least as good and sometimes even strictly better. Flexibility of choice translates such weak dominance relations in the above Savage-framework into preferences over opportunity sets. 
When preferences over opportunity sets satisfy strong ambiguity aversion and flexibility of choice the decision situation of our example reduces to the following candidates for preference-maximizing opportunity sets (neglecting possible indifference with singleton-chains):

States of nature

$$
o \quad\{o, a\} \quad\{o, b\} \quad\{o, c\} \quad\{o, a, b\} \quad\{o, a, c\} \quad\{o, c, b\} \quad\{o, a, b, c\}
$$

Chosen opportunity sets

$\begin{array}{lllllllll}\{o, a, b\} & o & a & b & o & b & a & b & b \\ \{o, a, c\} & o & a & o & c & a & c & c & c\end{array}$

This reduced table also demonstrates that the Savage-framework is inappropriate to determine preference-maximizing opportunity sets in our model. Any decision theory - such as subjective expected utility theory, Savage (1954) or Choquet expected utility theory, e.g. Gilboa (1987) — that separates between the utility of consequences and decision weights referring to the likelihood of events stipulates that in any state different alternatives must be comparable. But the decision-maker of our example is not able to compare the consequences of his opportunity set choices when the realized state is $\{o, c, b\}$ or $\{o, a, b, c\}$. Hence, incompleteness of preferences, which is a central feature of our model, cannot be meaningfully captured by the Savage-framework.

In the next section we therefore present our proposal for a utility representation of preferences over chains, e.g., $\left(o \prec_{\mathcal{A}} a \prec_{\mathcal{A}} b\right)$ and $\left(o \prec_{\mathcal{A}} a \prec_{\mathcal{A}} c\right)$ which does not require any formation of beliefs or likelihood considerations about the realization of states.

\section{An additive representation result}

This section defines our main technical axiom, cancellation, cf. Kraft, Pratt, and Seidenberg (1959), Fishburn (1996), Conder and Slinko (2003), which guarantees an additive utility representation of preferences over totally ordered opportunity sets that have at least two members.

First, we proceed with further definitions:

- Define the multiset generating operator $T$ as follows: for all chains $\left(x_{1} \precsim \mathcal{A} \cdots \precsim \mathcal{A} x_{n}\right) \in$ $\mathcal{C}$

$$
T\left(x_{1} \precsim_{\mathcal{A}} \ldots \precsim_{\mathcal{A}} x_{n}\right):=\left\{o, x_{2}, x_{2}, \ldots, x_{n-1}, x_{n-1}, x_{n}\right\}
$$

That is, for every $\left(x_{1} \precsim_{\mathcal{A}} \ldots \precsim_{\mathcal{A}} x_{n}\right) \in \mathcal{C}, T\left(x_{1} \precsim_{\mathcal{A}} \ldots \precsim_{\mathcal{A}} x_{n}\right)$ is a special case of a multiset, in which the same element $x \in\left\{o, x_{2}, \ldots, x_{n-1}, x_{n}\right\}$ appears twice in $T\left(x_{1} \precsim_{\mathcal{A}} \ldots \precsim_{\mathcal{A}} x_{n}\right)$, if and only if, $x_{1} \precsim_{\mathcal{A}} x \precsim_{\mathcal{A}} x_{n}$ with $x \neq o, x_{n}$. 


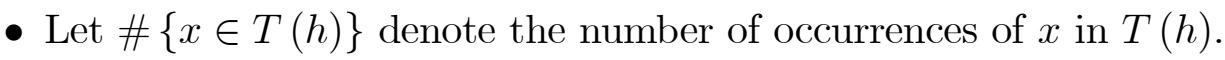

Definition: We say that the preference relation $\succeq$ over opportunity sets satisfies cancellation, if and only if, for every pair $h_{1}, h_{2}, \ldots, h_{m}$ and $g_{1}, g_{2}, \ldots, g_{m}$ of finite sequences of chains in $\mathcal{C}$ such that

$$
\sum_{j=1}^{m} \#\left\{x \in T\left(h_{j}\right)\right\}=\sum_{j=1}^{m} \#\left\{x \in T\left(g_{j}\right)\right\} \text { for all } x \in \mathcal{A},
$$

preferences $h_{j} \succeq g_{j}$ for all $j \in\{1, \ldots, m\}$ imply that $h_{j} \sim g_{j}$ for all $j \in\{1, \ldots, m\}$.

\section{Lemma:}

- If the preference relation $\succeq$ over opportunity sets satisfies cancellation and flexibility of choice, then preferences $\succeq$ over chains with at least two entries, i.e., members of $\mathcal{C}$, can be represented by a utility function $U: \mathcal{C} \rightarrow \mathbb{R}_{0}^{+}$ such that

$$
U\left(\left(x_{1} \precsim_{\mathcal{A}} \ldots \precsim_{\mathcal{A}} x_{n}\right)\right)=\sum_{k=1}^{n-1} f\left(x_{k}, x_{k+1}\right),
$$

where $f: \mathcal{A} \times \mathcal{A} \rightarrow \mathbb{R}_{0}^{+}$must satisfy the triangle-inequality:

$$
f(x, y)+f(y, z) \geq f(x, z)
$$

for all $x, y, z \in \mathcal{A}$.

- Conversely, if $f$ satisfies the triangle-inequality, then any function (1) represents preferences $\succeq$ over members of $\mathcal{C}$ such that the preference relation $\succeq$ over opportunity sets satisfies cancellation and flexibility of choice.

Proof of the lemma: Relegated to the appendix.

Corollary 1: If the preference relation $\succeq$ over opportunity sets satisfies cancellation, then preferences $\succeq$ over members of $\mathcal{C}$ also satisfy:

independence of connected chains: For all chains in $\mathcal{C}$ : 
- $\left(o \prec_{\mathcal{A}} a \precsim_{\mathcal{A}} \ldots \precsim_{\mathcal{A}} b\right),\left(o \prec_{\mathcal{A}} d \precsim_{\mathcal{A}} \ldots \precsim_{\mathcal{A}} e\right)$ and

$$
\begin{aligned}
&\left(o \prec_{\mathcal{A}} b \precsim_{\mathcal{A}} \ldots \precsim_{\mathcal{A}} c\right) \sim\left(o \prec_{\mathcal{A}} e \precsim_{\mathcal{A}} \ldots \precsim_{\mathcal{A}} f\right)\left(o \prec_{\mathcal{A}} b\right) \sim\left(o \prec_{\mathcal{A}} e\right) \\
&\left(o \prec_{\mathcal{A}} a \precsim_{\mathcal{A}} \ldots \precsim_{\mathcal{A}} b\right) \succeq\left(o \prec_{\mathcal{A}} d \precsim_{\mathcal{A}} \ldots \precsim_{\mathcal{A}} e\right) \text { iff } \\
&\left(o \prec_{\mathcal{A}} a \precsim_{\mathcal{A}} \ldots \precsim_{\mathcal{A}} b \precsim_{\mathcal{A}} \ldots \precsim_{\mathcal{A}} c\right) \succeq\left(o \prec_{\mathcal{A}} d \precsim_{\mathcal{A}} \ldots \precsim_{\mathcal{A}} e \precsim_{\mathcal{A}} \ldots \precsim_{\mathcal{A}} f\right) .
\end{aligned}
$$

- $\left(o \prec_{\mathcal{A}} a \precsim_{\mathcal{A}} \ldots \precsim_{\mathcal{A}} b\right),\left(o \prec_{\mathcal{A}} e \precsim_{\mathcal{A}} \ldots \precsim_{\mathcal{A}} f\right)$ and

$$
\begin{gathered}
\left(o \prec_{\mathcal{A}} b \precsim_{\mathcal{A}} \ldots \precsim_{\mathcal{A}} c\right) \sim\left(o \prec_{\mathcal{A}} d \precsim_{\mathcal{A}} \ldots \precsim_{\mathcal{A}} e\right) \\
\left(o \prec_{\mathcal{A}} b\right) \sim\left(o \prec_{\mathcal{A}} e\right) \\
\left(o \prec_{\mathcal{A}} a \precsim_{\mathcal{A}} \ldots \precsim_{\mathcal{A}} b\right) \succeq\left(o \prec_{\mathcal{A}} d \precsim_{\mathcal{A}} \ldots \precsim_{\mathcal{A}} e\right) \text { iff } \\
\left(o \prec_{\mathcal{A}} a \precsim_{\mathcal{A}} \ldots \precsim_{\mathcal{A}} b \precsim_{\mathcal{A}} \ldots \precsim_{\mathcal{A}} c\right) \succeq\left(o \prec_{\mathcal{A}} d \precsim_{\mathcal{A}} \ldots \precsim_{\mathcal{A}} e \precsim_{\mathcal{A}} \ldots \precsim_{\mathcal{A}} f\right) .
\end{gathered}
$$

Proof of corollary 1: Relegated to the appendix.

Corollary 2: Consider a preference relation $\succeq$ over opportunity sets which satisfies cancellation and flexibility of choice. Then the following two statements are equivalent:

(i) for any chain $h$ and any $a, a^{\prime} \in \mathcal{A}$ such that

$$
\begin{aligned}
h \cup\{a\} & \in \mathcal{C} \\
h \cup\left\{a^{\prime}\right\} & \in \mathcal{C} \\
h \cup\{a\} & \sim h \cup\left\{a^{\prime}\right\}
\end{aligned}
$$

and $h \succ h^{\prime}$ for all $h, h^{\prime} \in \mathcal{C}$ with $h^{\prime} \subset \subset h$.

(ii) The preference relation $\succeq$ over opportunity sets in $\mathcal{C}$ can be represented by (1) where $f$ is the discrete metric, i.e., for all $x, y \in \mathcal{A}, f(x, y)=1$ if $x \neq y$ and $f(x, y)=0$ otherwise.

Proof of corollary 2: The proof is straightforward and therefore left to the reader. (Proceed by induction over the number of entries and observe that if (1) represents 
preferences on $\mathcal{C}$ where $f$ is the discrete metric, then $h \succ(\sim) h^{\prime}$ for all $h, h^{\prime} \in \mathcal{C}$ iff $h$ has strictly more (equally many) entries than (as) $h^{\prime}$.)

Remark. The assumption of cancellation is closely related to the independence principle (or to Savage's, (1954) sure thing principle within the context of deriving additive probability measures). Since we are not convinced by the appeal (descriptive as well as normative) of the independence principle, we have chosen a formulation of cancellation which gives the convenient additive utility representation (1) while it admits for specific weakening of the independence principle at the same time. For example, if we had alternatively defined cancellation with respect to the number of occurrences of alternatives $x$ in the chains $h_{j}$ and $g_{j}$ in $\mathcal{C}$, the following preferences over opportunity sets would be excluded

$$
\begin{aligned}
\left(o \prec_{\mathcal{A}} a_{1} \precsim_{\mathcal{A}} a_{2}\right) & \succ\left(o \prec_{\mathcal{A}} b_{1} \precsim_{\mathcal{A}} b_{2}\right) \\
\left(o \prec_{\mathcal{A}} a_{3} \precsim_{\mathcal{A}} a_{4}\right) & \succeq\left(o \prec_{\mathcal{A}} b_{3} \precsim_{\mathcal{A}} b_{4}\right) \\
\left(o \prec_{\mathcal{A}} a_{3}\right) & \sim\left(o \prec_{\mathcal{A}} b_{3}\right) \\
\left(o \prec_{\mathcal{A}} b_{1} \precsim_{\mathcal{A}} b_{2} \precsim_{\mathcal{A}} b_{3} \precsim_{\mathcal{A}} b_{4}\right) & \succeq\left(o \prec_{\mathcal{A}} a_{1} \precsim_{\mathcal{A}} a_{2} \precsim_{\mathcal{A}} a_{3} \precsim_{\mathcal{A}} a_{4}\right)
\end{aligned}
$$

However, preferences (6a)-(6d) do not violate our concept of cancellation since it refers to the number of occurrences of alternatives $x$ in the multisets $T\left(h_{j}\right)$ and $T\left(g_{j}\right)$, for $j \in\{1, \ldots, m\}$. Preferences (6a)-(6d) are, therefore, representable by (1) as follows:

$$
\begin{aligned}
f\left(o, a_{1}\right)+f\left(a_{1}, a_{2}\right) & >f\left(o, b_{1}\right)+f\left(b_{1}, b_{2}\right) \\
f\left(o, a_{3}\right)+f\left(a_{3}, a_{4}\right) & \geq f\left(o, b_{3}\right)+f\left(b_{3}, b_{4}\right) \\
f\left(o, a_{3}\right) & =f\left(o, b_{3}\right) \\
f\left(o, b_{1}\right)+f\left(b_{1}, b_{2}\right)+f\left(b_{2}, b_{3}\right)+f\left(b_{3}, b_{4}\right) & \geq f\left(o, a_{1}\right)+f\left(a_{1}, a_{2}\right)+f\left(a_{2}, a_{3}\right)+f\left(a_{3}, a_{4}\right)
\end{aligned}
$$

Clearly, this system of inequalities holds for a sufficiently large difference $f\left(b_{2}, b_{3}\right)-$ $f\left(a_{2}, a_{3}\right)$.

Thus, while our concept of cancellation implies the independence principle for connected chains (cf. corollary 1), it does not entail the independence principle for arbitrary chains. In our framework, the evaluation of, e.g., the opportunity set

$$
\left(o \prec_{\mathcal{A}} a_{1} \precsim \mathcal{A} a_{2} \precsim \mathcal{A} a_{3} \precsim \mathcal{A} a_{4}\right)
$$

is not separable into evaluations of opportunity sets $\left(o \prec_{\mathcal{A}} a_{1} \precsim_{\mathcal{A}} a_{2}\right)$ and $\left(o \prec_{\mathcal{A}} a_{3} \precsim_{\mathcal{A}} a_{4}\right)$ because we allow for the possibility that the joint appearance of $a_{2}$ and $a_{3}$ in the opportunity set $\left(o \prec_{\mathcal{A}} a_{1} \precsim_{\mathcal{A}} a_{2} \precsim_{\mathcal{A}} a_{3} \precsim_{\mathcal{A}} a_{4}\right)$ might have some influence on the overall evaluation of this opportunity set. We do not necessarily believe that this weakening of 
the independence principle is the most sensible one, and we consider it a topic for future research to look for alternative approaches. Nevertheless, we think that our concept of cancellation has, firstly, the pragmatic advantage that it stands for a, in our opinion, desirable generalization of the independence principle and, secondly, it also admits for the very convenient utility representation as derived in the above lemma.

\section{$5 \quad$ Preference maximizing opportunity sets}

In this section, we derive our main result, which characterizes the opportunity sets that a welfare-maximizing social planner would like to implement. Before we present the according characterization theorem, recall that a function $\delta: \mathcal{A} \times \mathcal{A} \rightarrow \mathbb{R}_{0}^{+}$is a metric on $\mathcal{A}$, if and only if, $\delta$ satisfies the following three properties for all $a, b, c \in \mathcal{A}$ :

1. $a=b$ implies $\delta(a, b)=0$ and $a \neq b$ implies $\delta(a, b)>0$

2. $\delta(a, b)=\delta(b, a)$

3. $\delta(a, b)+\delta(b, c) \geq \delta(a, c)$ (triangle-inequality)

The following result identifies preference-maximizing opportunity sets as maximizers of a utility function which sums up the distances of its ordered elements for some appropriate metric imposed on all possible choices.

\section{A characterization theorem for preference maximizing opportunity sets:}

- If the preference relation $\succeq$ over opportunity sets satisfies strong ambiguity aversion, cancellation, and flexibility of choice, then an opportunity set $D \in \mathcal{D}_{o}$ maximizes the preference relation $\succeq$ over opportunity sets, if and only if, $D:=\left(o \prec_{\mathcal{A}} a \precsim_{\mathcal{A}} \ldots \precsim_{\mathcal{A}} b\right) \in \mathcal{C}$ such that for some metric $\delta$ on $\mathcal{A}$ :

$$
\left(o \prec_{\mathcal{A}} a \precsim_{\mathcal{A}} \cdots \precsim_{\mathcal{A}} b\right) \in \arg \max _{\left(x_{1} \precsim \mathcal{A} \cdots \precsim \mathcal{A} x_{n}\right) \in \mathcal{C}} \sum_{k=1}^{n-1} \delta\left(x_{k}, x_{k+1}\right)
$$

- If a chain $\left(o \prec_{\mathcal{A}} a \precsim_{\mathcal{A}} \ldots \precsim_{\mathcal{A}} b\right) \in \mathcal{C}$ maximizes the r.h.s. of (7) for an arbitrary metric $\delta$ on $\mathcal{A}$ then $\left(o \prec_{\mathcal{A}} a \precsim_{\mathcal{A}} \ldots \precsim_{\mathcal{A}} b\right)$ maximizes some preferences $\succeq$ over opportunity sets satisfying strong ambiguity aversion, flexibility of choice and cancellation.

Proof: At first observe that, by strong ambiguity aversion, any preference maximizing opportunity set must be some chain: taking away alternatives from the opportunity set is strictly preferred as long as not all alternatives are comparable, i.e., as long as the 
remaining alternatives are not some chain. Moreover, by assumption 3 and flexibility of choice, there exist non-degenerate chains which are preference maximizing. The characterization theorem then follows from the lemma, since any function $f$ in (1), satisfying the triangle-inequality, can be extended to some metric $\delta$ on $\mathcal{A}$. $\square$

Corollary 3: Consider a preference relation $\succeq$ over opportunity sets which satisfies strong ambiguity aversion, cancellation, and flexibility of choice. Suppose further that the following assumptions are fulfilled:

- for any chain $h \in C$ and any $a, a^{\prime} \in \mathcal{A}$ such that

$$
\begin{aligned}
h \cup\{a\} & \in \mathcal{C} \\
h \cup\left\{a^{\prime}\right\} & \in \mathcal{C} \\
h \cup\{a\} & \sim h \cup\left\{a^{\prime}\right\}
\end{aligned}
$$

and $h \succ h^{\prime}$ for all $h, h^{\prime} \in \mathcal{C}$ with $h^{\prime} \subset \subset h$.

Then an opportunity set $D \in \mathcal{D}_{\text {o }}$ maximizes the preference relation $\succeq$ over opportunity sets, if and only if, $D:=\left(o \prec_{\mathcal{A}} a \precsim_{\mathcal{A}} \cdots \precsim_{\mathcal{A}} b\right) \in \mathcal{C}$ and there does not exist $a$ chain $h \in \mathcal{C}$ such that $h$ has strictly more entries than $D$.

Proof: Since statement (i) of corollary 2 is satisfied, $\delta$ denotes in (7) the discrete metric so that for all $\left(x_{1} \precsim \mathcal{A} \ldots \precsim \mathcal{A} x_{n}\right) \in \mathcal{C}$

$$
\sum_{k=1}^{n-1} \delta\left(x_{k}, x_{k+1}\right)=n-1
$$

Thus, the utility of opportunity sets in $\mathcal{C}$ is strictly increasing in the number of alternatives contained in these chains, whereas the decision-maker is indifferent between any two non-degenerate chains containing the same number of alternatives.

\section{An illustrative example: Ambiguity arising from incomparable features}

In this section, we show that strong ambiguity aversion does not put severe restrictions on actually observed behavior. In particular, we demonstrate how orderings on $\mathcal{A}$ which differ with respect to their incomplete parts can generate different preference-maximizing 
opportunity sets. Intuitively, the more incomparable pairs of alternatives the set $\mathcal{A}$ contains, the fewer elements are included in the preference maximizing set.

In the following, we focus on a specific class of preferences over alternatives such that all alternatives exhibit a comparable as well as an incomparable component from the decision-maker's point of view.

Assumption 4: Let $\mathcal{A} \subset \Lambda \times \Gamma \cup\{o\}$ such that $o \in \mathcal{A}$ and suppose that the preference relation $\precsim \mathcal{A}$ is complete on $\Lambda \times\{\gamma\} \cup\{o\} \cap \mathcal{A}$, for any $\gamma \in \Gamma$.

We will interpret $\lambda \in \Lambda$, respectively $\gamma \in \Gamma$, as the comparable, respectively incomparable, component of the alternative $(\lambda, \gamma) \in \mathcal{A}$. Accordingly, we will say that $\Lambda$ collects the comparable features whereas $\Gamma$ collects the incomparable features of the alternatives in $\mathcal{A}$. While the decision-maker can, by assumption 4 , easily compare alternatives that share identical incomparable features, he cannot easily choose between alternatives having different incomparable features.

Recall our initial example of a college graduate applying for a $\mathrm{PhD}$ program whereby:

$$
\begin{aligned}
& \Lambda:=\text { prestige } \times \text { tuition } \times \text { scholarship } \\
& \Gamma:=\text { pressure to perform } .
\end{aligned}
$$

Thus, such a student can easily compare different values of prestige, tuition and scholarship but he encounters severe problems to compare different degrees of pressure to perform.

In what follows we present two benchmark cases where different incomparable features result in incomplete preferences over alternatives.

\section{Case I: Weak ambiguity perception}

Definition: We say that $\precsim_{\mathcal{A}}$ expresses weak ambiguity perception if, for all $(\lambda, \gamma),\left(\lambda^{\prime}, \gamma^{\prime}\right) \in$ $\mathcal{A},(\lambda, \gamma) A\left(\lambda^{\prime}, \gamma^{\prime}\right)$ if and only if $\lambda=\lambda^{\prime}$ and $\gamma \neq \gamma^{\prime}$.

(Example continued.) Suppose a graduate student, exhibiting weak ambiguity perception, has to decide between the $\mathrm{PhD}$ programs offered by Cornell and by Duke, respectively. For the sake of argument, assume that the 
student perceives the comparable features - prestige, tuition, scholarship of both programs as identical whereas he expects less pressure to perform under the Cornell than under the Duke program. Since, by assumption, he cannot make up his mind whether a low or a high pressure to perform would suit him better, he is caught in an ambiguous choice situation. On the other hand, if he is confronted with the choice between Cornell offering a higher scholarship than Duke - with otherwise identical specifications of the comparable features - he would have no difficulties to decide for the Cornell $\mathrm{PhD}$ program.

Obviously, if a decision-maker expresses weak ambiguity perception, and the comparable features $\lambda \in \Lambda$ were different for all alternatives $(\lambda, \gamma) \in \mathcal{A}$, then $\mathcal{A}$ itself would be a chain and therefore the unique maximizer of (7) for any distance function $\delta$ on $\mathcal{A} \times \mathcal{A}$.

(Example continued.) If every $\mathrm{PhD}$ program offered distinct comparable features $\lambda$, a graduate student with weak ambiguity perception would prefer to apply to all $\mathrm{PhD}$ programs.

\section{Case II: Strong ambiguity perception}

Definition: We say that $\precsim_{\mathcal{A}}$ expresses strong ambiguity perception if, for all $(\lambda, \gamma),\left(\lambda^{\prime}, \gamma^{\prime}\right) \in$ $\mathcal{A},(\lambda, \gamma) A\left(\lambda^{\prime}, \gamma^{\prime}\right)$ if and only if $\gamma \neq \gamma^{\prime}$.

(Example continued.) In contrast to weak ambiguity perception, strong ambiguity perception additionally requires that the graduate student cannot compare $\mathrm{PhD}$ programs with different specifications of prestige, tuition and scholarship whenever they include different levels of the incomparable feature pressure to perform.

Under the assumptions of our representation theorem, the possible candidates for a preference maximizing opportunity set are given as the chains

$$
\Lambda \times\{\gamma\}, \gamma \in \Gamma
$$

That is, (i) for any $\gamma \in \Gamma$ we can find some distance function $\delta$ on $\mathcal{A} \times \mathcal{A}$ such that $\Lambda \times\{\gamma\}$ is preference-maximizing and (ii) for any given distance function $\delta$ on $\mathcal{A} \times \mathcal{A}$ only some set $\Lambda \times\{\gamma\}$ with $\gamma \in \Gamma$ will be a maximizer of (7).

(Example continued.) A graduate student with strong ambiguity perception prefers to apply to multiple $\mathrm{PhD}$ programs given that they impose an identical pressure to perform. For a given $\gamma \in \Gamma$ suppose that 
$\left(\lambda_{i(\gamma)}, \gamma\right) \precsim \mathcal{A}\left(\lambda_{i(\gamma)+1}, \gamma\right), i(\gamma) \in \mathbb{N}$, for all $\lambda_{i(\gamma)}, \lambda_{i(\gamma)+1} \in \Lambda, \lambda_{i(\gamma)} \neq \lambda_{i(\gamma)+1}$.

He will then choose the level of pressure to perform $\gamma$ in such a way that

$$
\begin{gathered}
\delta\left(o,\left(\lambda_{i(\gamma)}, \gamma\right)\right)+\sum_{i=1}^{\# \Lambda-1} \delta\left(\left(\lambda_{i(\gamma)}, \gamma\right),\left(\lambda_{i(\gamma)+1}, \gamma\right)\right) \\
\geq \delta\left(o,\left(\lambda_{i\left(\gamma^{\prime}\right)}, \gamma^{\prime}\right)\right)+\sum_{i=1}^{\# \Lambda-1} \delta\left(\left(\lambda_{i\left(\gamma^{\prime}\right)}, \gamma^{\prime}\right),\left(\lambda_{i\left(\gamma^{\prime}\right)+1}, \gamma^{\prime}\right)\right)
\end{gathered}
$$

holds for all $\gamma^{\prime} \in \Gamma$, where $\lambda_{i(\gamma)}$ and $\lambda_{i(\gamma)+1}$ denote different comparable features of the $\mathrm{PhD}$ programs.

We could obtain similar results by keeping the ordering $\precsim_{\mathcal{A}}$ constant, while varying the attitude of the decision-maker towards ambiguity. This alternative approach, however, presents a problem. If a decision-maker expresses the preference:

$$
D \cup\{a\} \succ D
$$

for some $a \notin D$, we would not know whether this is due to the fact that $a$ is comparable to all elements in $D$ or whether the decision-maker finds that the utility derived from adding $a$ to the set compensates for the ambiguity resulting from $a$ being incomparable to some elements of $D$. As long as we do not think of incomparability as an objective property, it will be impossible to disentangle these two effects without using strong additional assumptions on preferences. In contrast, the assumption of strong ambiguity aversion allows us to clearly separate the effects of flexibility and ambiguity and to uniquely identify the incomplete part of $\precsim_{\mathcal{A}}$ by observing choices between menus.

\section{Concluding remarks and outlook}

This paper describes the optimization problem of a social planner who would like to maximize the welfare of decision-makers that dislike ambiguous choice situations. We identify conditions on preferences which imply that optimal opportunity sets contain only comparable alternatives and are maximal with respect to this property. As our main formal result we characterize the preference-maximizing opportunity set as a maximizer of a utility function which sums up the distances of its ordered elements for some appropriate metric imposed on all alternatives.

Our results are driven by the assumption of strong aversion towards ambiguity. We are far from insisting that this assumption describes a behavioral pattern which is most prevalent in practice. We argue, however, that it captures the general idea that people are averse to making choices between incomparable alternatives. We consider this 
benchmark case as a first step towards developing a more general theory of ambiguity aversion, in which a range of attitudes towards incomparable alternatives could be modelled. The assumption of strong ambiguity aversion by far simplifies our analysis and allows us to draw conclusions about preference maximizing opportunity sets which would not be valid under different assumptions. Interestingly enough, we find that the conclusions which we reach can be supported by institutions found in everyday life.

While the scope of this paper is restricted to the characterization of welfare-maximizing opportunity sets, it would be compelling — from a decision-theoretic point of view — to derive a complete functional representation of preferences over opportunity sets. Such a representation would enable us to model trade-offs between preferences for flexibility on chains and ambiguity aversion with respect to incomparable alternatives.

To see how such trade-offs naturally emerge in our model, let us once again return to the example we presented in section 3. Suppose $x_{2} \precsim_{\mathcal{A}} \ldots \precsim_{\mathcal{A}} x_{50}$ and $y \prec_{\mathcal{A}} x_{j}$ for $j \in\{3, \ldots, 50\}$ whereas $x_{2} A y$. In general, the decision-maker will have to trade-off the additional flexibility obtained by adding $y$ to the set versus its incomparability to $x_{2}$. If $y$ is added to the set, a new maximal chain, $\left(o, y, x_{3}, \ldots, x_{50}\right)$, emerges. Note that this new maximal chain coincides with the old one on the set $\left(x_{3} \ldots x_{50}\right)$. Hence, it seems intuitive to measure the additional flexibility by the subjective length of the chain $\left(o, y, x_{3}\right)$. If this chain is sufficiently long according to $\delta$, the additional flexibility introduced by adding $y$ will compensate for the ambiguity resulting from the incomparability between $y$ and $x_{2}$.

Note that we measure the subjectively perceived ambiguity implicitly by the amount of flexibility necessary to compensate for it. Indeed, the length of the newly added chain $\left(o, y, x_{3}\right)$ necessary to make the decision-maker indifferent between

$$
\begin{aligned}
& \left\{o, x_{2} \ldots x_{50}\right\} \text { and } \\
& \left\{o, y, x_{2} \ldots x_{50}\right\}
\end{aligned}
$$

can serve as a measure of ambiguity-aversion of the decision-maker. Especially, for an ambiguity-neutral decision-maker this length will be 0. Such a person will express preferences

$$
D \cup\{x\} \succsim D
$$

irrespectively of the number of elements in $D$ deemed incomparable to $x$. In contrast, for the strongly ambiguity averse decision-maker of our present approach, the length necessary to compensate for the additional ambiguity will be larger - according to the decision-maker's subjective metric $\delta$ - than that of the longest chain in $\mathcal{A}$.

In terms of our analysis, both ambiguity neutrality and strong aversion towards ambiguity seem to represent rather extreme cases. It would be interesting to come up 
with a system of axioms which would allow to capture the whole scale of attitude towards ambiguity ranging from the ambiguity neutral to the strongly ambiguity averse decisionmaker. We intend to address these decision-theoretic issues in subsequent research.

\section{Appendix}

\section{Proof of the lemma:}

First, note that cancellation is a sufficient condition (e.g., Fishburn, 1996) for the existence of a utility representation $V$ of a complete pre-ordering $\gtrsim$ on the set $\Gamma:=$ $\{T(h) \mid h \in \mathcal{C}\}$ such that there exist utility numbers $u: \mathcal{A} \rightarrow \mathbb{R}$ with

$$
V\left(\left\{x_{1} \equiv o, x_{2}, x_{2}, \ldots, x_{n-1}, x_{n-1}, x_{n}\right\}\right):=\sum_{j=1}^{n} u\left(x_{j}\right)+\sum_{j=2}^{n-1} u\left(x_{j}\right)
$$

flexibility of choice further implies that these numbers can be chosen to be non-negative since the addition of a further element to a chain should weakly increase its evaluation. Now define the function $f\left(x_{j}, x_{j+1}\right):=u\left(x_{j}\right)+u\left(x_{j+1}\right)$ for all $j \in\{1, \ldots, n-1\}$. Let $h \sim$ $(\succ) h^{\prime}$ iff $T(h) \sim(>)\left(T\left(h^{\prime}\right)\right)$ for all $h, h^{\prime} \in \mathcal{C}$ so that the sets $\mathcal{C}$ and $\Gamma$ are homeomorphic whereby, (8) becomes (1). In order to see that any such function $f$ must satisfy the triangle-inequality, observe that

$$
\begin{aligned}
V(\{o, a, c\}) & =u(o)+u(a)+u(a)+u(c) \\
& \leq u(o)+u(a)+u(a)+u(b)+u(b)+u(c)=V(\{o, a, a, b, b, c\}) \Leftrightarrow \\
U\left(\left(o \prec_{\mathcal{A}} a \precsim{ }_{\mathcal{A}} c\right)\right) & =f(o, a)+f(a, c) \\
& \leq f(o, a)+f(a, b)+f(b, c)=U\left(\left(o \prec_{\mathcal{A}} a \precsim_{\mathcal{A}} b \precsim_{\mathcal{A}} c\right)\right) \Leftrightarrow \\
f(a, c) & \leq f(a, b)+f(b, c) .
\end{aligned}
$$

Which proves the first part of the lemma.

Since cancellation and flexibility of choice (e.g., Fishburn, 1996) are also necessary conditions for an additive utility representation (8), the second part of the lemma easily follows. For a given function $f$, let

$$
\begin{aligned}
u\left(x_{1}\right): & =f\left(x_{1}, x_{2}\right)-u\left(x_{2}\right) \\
& \cdots \\
u\left(x_{n-1}\right): & =f\left(x_{n-1}, x_{n}\right)-u\left(x_{n}\right)
\end{aligned}
$$

Just choose some non-negative $u\left(x_{n}\right) \leq f\left(x_{n-1}, x_{n}\right)$, so that the remaining utility numbers $u\left(x_{j}\right)$ are uniquely determined for all $j \in\{1, \ldots, n-1\}$. Observe that we have for all $x \precsim_{\mathcal{A}} y \precsim_{\mathcal{A}} z$

$$
f(x, z)=f(x, y)+f(y, z)-2 \cdot u(y)
$$


Thus, if $f$ satisfies the triangle-inequality, then $u\left(x_{j}\right) \geq 0$ for all $j \in\{1, \ldots, n\}$. As a consequence, (1) becomes a representation (8) of preferences that satisfy cancellation and flexibility of choice.

Proof of corollary 1: The utility representation (1) implies for all $\left(o \prec_{\mathcal{A}} a \precsim_{\mathcal{A}} \ldots \precsim_{\mathcal{A}} b\right)$, $\left(o \prec_{\mathcal{A}} d \precsim_{\mathcal{A}} \ldots \precsim_{\mathcal{A}} e\right)$ satisfying

$$
\begin{aligned}
\left(o \prec_{\mathcal{A}} b \precsim_{\mathcal{A}} \ldots \precsim_{\mathcal{A}} c\right) & \sim\left(o \prec_{\mathcal{A}} e \precsim_{\mathcal{A}} \ldots \precsim_{\mathcal{A}} f\right) \\
\left(o \prec_{\mathcal{A}} b\right) & \sim\left(o \prec_{\mathcal{A}} e\right)
\end{aligned}
$$

the equalities

$$
\begin{aligned}
U\left(o \prec_{\mathcal{A}} b \precsim_{\mathcal{A}} \ldots \precsim_{\mathcal{A}} c\right) & =U\left(o \prec_{\mathcal{A}} e \precsim_{\mathcal{A}} \ldots \precsim_{\mathcal{A}} f\right) \\
U\left(o \prec_{\mathcal{A}} b\right) & =U\left(o \prec_{\mathcal{A}} e\right) .
\end{aligned}
$$

Hence,

$$
\begin{aligned}
U\left(\left(o \prec_{\mathcal{A}} a \precsim_{\mathcal{A}} \ldots \precsim_{\mathcal{A}} b \precsim_{\mathcal{A}} \ldots \precsim_{\mathcal{A}} c\right)\right)= & U\left(o \prec_{\mathcal{A}} a \precsim_{\mathcal{A}} \ldots \precsim_{\mathcal{A}} b\right)+U\left(o \prec_{\mathcal{A}} b \precsim_{\mathcal{A}} \ldots \precsim_{\mathcal{A}} c\right) \\
& -U\left(o \prec_{\mathcal{A}} b\right) \\
= & U\left(o \prec_{\mathcal{A}} a \precsim_{\mathcal{A}} \ldots \precsim_{\mathcal{A}} b\right)+U\left(o \prec_{\mathcal{A}} e \precsim_{\mathcal{A}} \ldots \precsim_{\mathcal{A}} f\right) \\
& -U\left(o \prec_{\mathcal{A}} e\right) \\
\geq & U\left(o \prec_{\mathcal{A}} d \precsim_{\mathcal{A}} \ldots \precsim_{\mathcal{A}} e\right)+U\left(o \prec_{\mathcal{A}} e \precsim_{\mathcal{A}} \ldots \precsim_{\mathcal{A}} f\right) \\
& -U\left(o \prec_{\mathcal{A}} e\right),
\end{aligned}
$$

iff

$$
\begin{aligned}
U\left(o \prec_{\mathcal{A}} a \precsim_{\mathcal{A}} \ldots \precsim_{\mathcal{A}} b\right) & \geq U\left(o \prec_{\mathcal{A}} d \precsim_{\mathcal{A}} \ldots \precsim_{\mathcal{A}} e\right), \text { or } \\
\left(o \prec_{\mathcal{A}} a \precsim_{\mathcal{A}} \ldots \precsim_{\mathcal{A}} b\right) & \succeq\left(o \prec_{\mathcal{A}} d \precsim_{\mathcal{A}} \ldots \precsim_{\mathcal{A}} e\right) .
\end{aligned}
$$

Proceed analogously to prove the equivalence of (4) and (5). Note that flexibility of choice is not needed for the additive representation, but only insures that the utility numbers $u\left(x_{j}\right)$ defined in the proof of Lemma 1 are positive, a fact that is not used in the proof of the corollary. $\square$ 


\section{References}

Conder, N. and A. Slinko (2003), "A counterexample to Fishburn's conjecture", University of Auckland, mimeo.

Danan, E. and A. Ziegelmeyer (2004), "Are prefereneces incomplete? An experimental study using flexible choices", Discussion Papers on Strategic Interaction 23-2004.

Deci, E.L. (1975), Intrinsic motivation, Plenum Press, New York.

Deci, E.L. (1981), The psychology of self-determination, Heath, Lexington, MA.

Deci, E.L. and R.M. Ryan (1985), Intrinsic motivation and self-determination in human behavior, Plenum Press, New York.

Dekel, E., Lipman, B.L., and A. Rustichini (2001), "Representing preferences with a unique subjective state space", Econometrica 69, 891-934.

Dekel, E., Lipman, B.L., and A. Rustichini (2004), "Temptation-driven preferences", Boston University, mimeo.

Eliaz, K. and E. Ok (2006), "Indifference or indecisiveness", Games and Economic Behavior 25, 61-86.

Fishburn, P.C. (1996), "Finite linear qualitative probability", Journal of Mathematical Psychology 40, 64-77.

Gale, D. and L. S. Shapley (1962), College Admissions and the Stability of Marriage, American Mathematics Monthly 69, 9-15.

Gilboa, I. (1987), "Expected utility with purely subjective non-additive probabilities", Journal of Mathematical Economics 16, 65-88.

Gul, F. and W. Pesendorfer (2001), "Self-control and the theory of consumption", Econometrica 72, 119-158.

Iyengar, S.S. and M. Lepper (2000), "When choice is demotivating: Can one desire too much of a good thing?", Journal of Personality and Social Psychology, 79, 995-1006.

Iyengar, S.S. and M.R. Lepper (2002), "Choice and its consequences: On the costs and benefits of self-determination", in: Tesser, A. (Ed.) Self and motivation: emerging psychological perspectives, 71-96, American Psychological Association, Washington D.C. 
Iyengar, S.S., Jiang, W., and G. Huberman (2004), "How much choice is too much: Determinants of individual contributions in 401K retirement plans", in: Mitchell, O.S. \& Utkus, S. (Eds.) Pension design and structure: New lessons from behavioral finance, 83-97, Oxford University Press, Oxford.

Kahneman, D. and A. Tversky (1984), "Choices, values, and frames", American Psychologist 39, 341-350.

Karni, E. and Z. Safra (1990), "Behaviorally consistent optimal stopping rules", Journal of Economic Theory 519, 391-402.

Kraft, C.H., Pratt, J.W., and A. Seidenberg (1959), "Intuitive probability on finite sets", Annals of Mathematical Statistics 30, 408-419.

Kreps, D.M. (1979), "A representation theorem for "preferences for flexibility"”, Econometrica 47, 565-578.

Laibson, D. (1997), "Golden eggs and hyperbolic discounting", Quarterly Journal of Economics 112, 443-477.

Ludwig, A., and A. Zimper (2006), "Investment behavior under ambiguity. The case of pessimistic decision makers", Mathematical Social Sciences, forthcoming.

Nehring, K. (1999), "Preferences for flexibility in a Savage framework", Econometrica 67, 101-119.

Savage, L.J. (1954), The foundations of statistics, John Wiley and \& Sons, Inc.: New York, London, Sydney.

Shafir, E., Simonson, I., and A. Tversky (1993), "Reason-based choice", Cognition 49, 11-36.

Strotz, R.H. (1956), "Myopia and inconsistency in dynamic utility maximization", The Review of Economic Studies 23, 165-180.

Thaler, R.H. and C. R. Sunstein (2003), "Libertarian paternalism", American Economic Review 93, 175-179. 